University of Louisville

ThinkIR: The University of Louisville's Institutional Repository

$12-2013$

\title{
The effects of gender on anxiety sensitivity in relation to interoceptive task performances : an exploratory study.
}

Brittany Deanne Miller

University of Louisville

Follow this and additional works at: https://ir.library.louisville.edu/honors

Part of the Psychology Commons

\section{Recommended Citation}

Miller, Brittany Deanne, "The effects of gender on anxiety sensitivity in relation to interoceptive task performances : an exploratory study." (2013). College of Arts \& Sciences Senior Honors Theses. Paper 96. http://doi.org/10.18297/honors/96

This Senior Honors Thesis is brought to you for free and open access by the College of Arts \& Sciences at ThinkIR: The University of Louisville's Institutional Repository. It has been accepted for inclusion in College of Arts \& Sciences Senior Honors Theses by an authorized administrator of ThinkIR: The University of Louisville's Institutional Repository. This title appears here courtesy of the author, who has retained all other copyrights. For more information, please contact thinkir@louisville.edu. 
Running Head: The Effects of Gender on Anxiety Sensitivity in Relation to Interoceptive Tasks 1

The Effects of Gender on Anxiety Sensitivity in Relation to Interoceptive Task Performances: An Exploratory Study By Brittany Deanne Miller Submitted in partial fulfillment of the requirements for Graduation (summa or magna) cum laude and for graduation with honors from the Department of Psychological and Brian Sciences University of Louisville November, 2013 


\begin{abstract}
This study examined whether males or females have higher anxiety sensitivity, and how this may affect performance on interoceptive tasks. Females in this study, reported significantly higher rates of anxiety sensitivity than males, as measured by the Anxiety Sensitivity Index-3 (Taylor et al., 2007). No significant gender differences were found on either of two interoceptive sensitivity tasks, the Schandry Heartbeat Detection task (Schandry, 1981) and the Mindfulness Interoceptive Sampling Task (Dreeben, 2012). Results of this study suggest that, although females have higher self-reported anxiety sensitivity than males, this does not appear to either enhance or hinder their performance on interoceptive awareness tasks.
\end{abstract}




\section{TABLE OF CONTENTS}

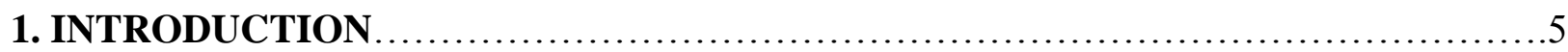

1.1 Gender Versus Sex.....................................................6

1.2 Defining Anxiety Sensitivity and Anxiety....................................

1.3 Understanding Interoception and Interoceptive Sensitivity $\ldots \ldots \ldots \ldots \ldots \ldots \ldots \ldots \ldots$

1.4 Mindfulness and Mindful Interoception...................................

1.5 Background Research................................................ 8

1.6 Hypotheses. ......................................................... 11

Table 1: Predicted Relationships Among Key Variables for Females............13

Table 2: Predicted Relationships Among Key Variables for Males..............13

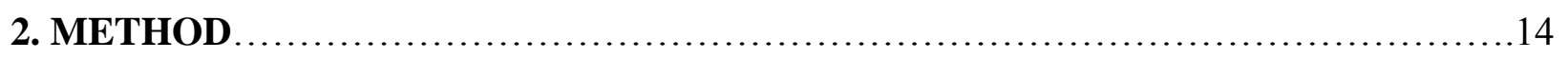

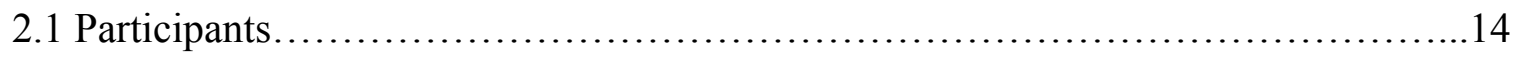

2.2 Materials............................................................. 14

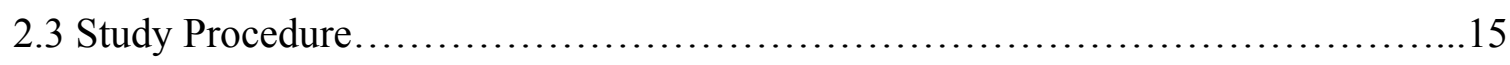

2.4 Schandry Protocol..................................................... 16

2.5 MIST Protocol...................................................... 17

Table 3: MIST Sequences........................................... 18 


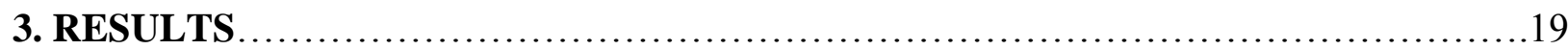

3.1 Preliminary Analysis........................................................ 19

3.2 Descriptive Statistics and Group Statistics.......................................19

Table 4: Summary Table of Descriptive Statistics...........................20

3.3 Independent Samples T-Test: ASI-3 ......................................20

3.4 Independent Samples T-Test: Schandry Task..................................21

3.5 Independent Samples T-Test: MIST .........................................21

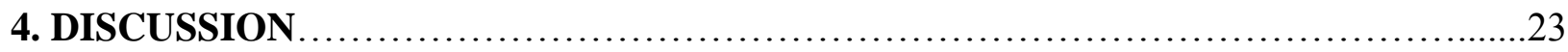

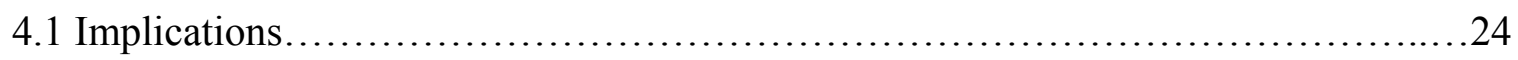

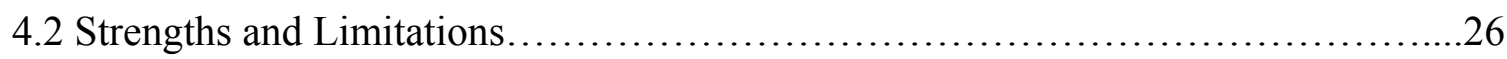

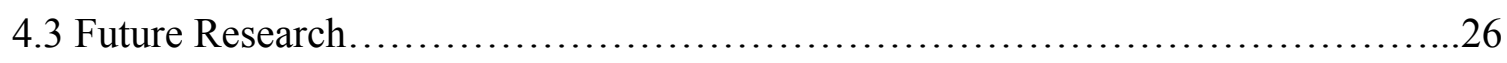

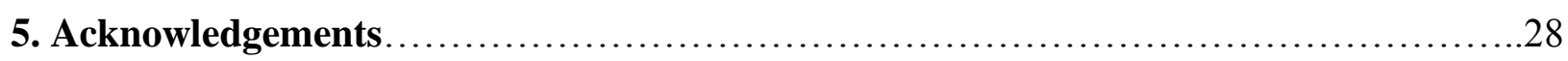

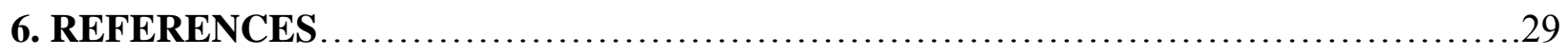

Appendix A: Anxiety Sensitivity Index-3 Questionnaire................................32

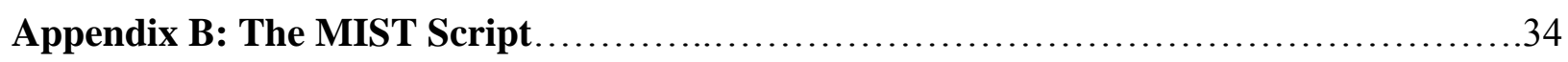




\section{INTRODUCTION}

Anxiety is a growing area of research and a growing problem world-wide; however, much about anxiety and anxiety disorders is not thoroughly understood in terms of causal factors and etiology (Doğan, 2012; Stoyanova \& Hope, 2012). Research examining anxiety and its relationship to awareness of inner sensations (interoception), for instance, is available; however, among that research, I found gender differences were sometimes overlooked. Within the research that does examine gender and anxiety is a study by Soysa and Wilcomb (2013), who explored whether gender, self-compassion, mindfulness, and self-efficacy are accurate predictors of anxiety, well-being, and depression; where they represented the importance of examining these particular predicting variables. For instance, they found that mindful non-judging inversely related to anxiety and gender was established as an accurate predictor of well-being (Soysa \& Wolcomb, 2013). Gender differences are important to study because they can help clarify possible differences between the sexes in interoceptive task performance and anxiety sensitivity. This study will serve as a gateway to determine whether there are differences in interoceptive awareness and anxiety sensitivity as a function of gender. Studying gender differences in anxiety sensitivity and how it relates to interoception merits further study because knowing who may be more prone to anxiety will allow researchers to target specific populations in order to understand what makes them more susceptible, and perhaps help reduce their anxiety. Also, studying gender differences across various interoceptive tasks may help explain relationships between anxiety sensitivity and inner body awareness. More specifically, if anxiety sensitivity and interoceptive task performance are related, researchers can use such information to provide possible explanations as to the mechanisms underlying the relationships. 


\subsection{Gender Versus Sex}

We have to first differentiate between the terms "gender" and "sex." Gender is often defined as the sociocultural differences (masculine versus feminine) between males and females, whereas sex is often defined in terms of biological differences (male versus female) (Reilly, 2012; Kimura, 2000; Muehlenhard \& Peterson, 2011). For the purposes of this paper, I will operationally refer to "gender differences" as the overall effects of both sociocultural and biological components on anxiety sensitivity and interoceptive task performance, although I did not examine in detail either sociocultural or biological aspects, I did view them as both being capable of causing effect on anxiety sensitivity and interoceptive performance.

\subsection{Defining Anxiety Sensitivity and Anxiety}

In this study, anxiety sensitivity was operationally defined as "the fear of arousal-related sensations, arising from beliefs that the sensations have adverse consequences such as death, insanity, or social rejection" (Taylor et al., 2007, p. 177; Reiss \& McNally, 1985). Furthermore, anxiety sensitivity is highly related to overall anxiety levels which can lead to a variety of anxiety disorders (Taylor et al. 2007, Reiss \& McNally, 1985; Taylor, 1999). "This is because AS [Anxiety Sensitivity] is an anxiety amplifier; when highly anxiety-sensitive people become anxious, they become alarmed about their arousal-related sensations, which further intensifies their anxiety" (Taylor et al., 2007, p.177). Therefore, by measuring anxiety sensitivity, I am measuring the likelihood of higher overall anxiety levels.

\subsection{Understanding Interoception and Interoceptive Sensitivity}

It is important to understand what is meant by the term, "interoception". Craig (2003) defines interoception as "perceive[d] [sensations] in the body that provide a sense of physical 
condition and underlie mood and emotional state" (p.500). He went on to describe interoception as "less distinct visceral [sensations] of vasomotor activity, hunger, thirst and internal sensations [which] are associated with a separate "interoceptive system" (Craig, 2003, p. 500).

Interoception, then, is operationally defined as internal bodily sensations, which can be perceived and interpreted by the observer. Furthermore, interoceptive sensitivity is operationally defined as measuring the degree in which people are aware of interoceptive cues (Ainley, TajaduraJiménez, Fotopoulou, \& Tsakiris, 2012). For example, one who is interoceptively sensitive may recognize internal sensations, such as their heart beating, more readily than someone who is less interoceptively sensitive.

\subsection{Mindfulness and Mindful Interoception}

Another important aspect of this study is the concept of "mindfulness". Though I did not test any hypotheses about the effects of anxiety overall or its effects on mindful interoceptive awareness, I looked at the relationships in an exploratory manner. The idea of "mindfulness" first originated in Buddhist communities where it was thought to result "in the development of wisdom, compassion, and ethics” (Kang \&Whittingham, 2010, p. 161). Contemporarily, mindfulness has been explained as the "capacity to avoid distraction" (Mrazek, Smallwood \& Schooler, 2012, p. 442). Furthermore, mindfulness can be used in meditation practices, such as described by Keune and Forintos (2010). For example, Keune and Forintos (2010) stated that "patients [can] engage in meditation exercises to refine their attentional skills and to learn to purposefully relate to the present moment experience in a non-judgmental manner" (p.373). So the overarching operational definition of mindfulness is non-judgmental attention (Kabat-Zinn, 2003; Keune \& Fortinos, 2010). Interoception, as noted earlier, is perceived sensations in the 
body (Craig, 2003). Therefore, mindful interoception can be defined as non-judgmental, uninterrupted, awareness of inner body sensations.

\subsection{Background Research}

Among the research that examines interoception and its relation to anxiety, is research by Schandry (1981). In this study, Schandry (1981) makes the argument that, "individuals who show [more accurate] perception of heart activity tend to exhibit higher levels of a momentarily experienced emotion (in this case anxiety)..." (p. 483). Schandry (1981) measured this

momentarily experienced emotion using a heart beat detection task, which throughout this paper will be referred to as, "the Schandry Task". In this task, the researcher connected the participants to an EKG sensor, which monitors cardiac activity including pulse or heart rate, and other devices to detect skin conductance, and respiration. In three different time intervals $(25,35$, and 45 seconds, which were unknown to the participant), the participants were told to count how many heart beats occurred within their body. They had to do this solely by concentrating on sensations from the heart. Furthermore, there were 30 second breaks between each time interval. Then, the reported number was compared (using percentage error) against the actual number measured by the EKG for each time interval, then the three intervals were summed. Higher scores yield less accurate perception and zero indicates perfectly accurate perception. In the study, conducted on 16 female subjects and 23 male subjects, Schandry (1981) found that although subjects had an increase in respiration, there was no change in heart rate. Schandry (1981) also found that those who were "good perceivers," (i.e. more accurate perceivers) reported higher levels of emotional experience and were higher on the personality trait, "emotional liability," than "poor perceivers" (i.e. less accurate perceivers), as measured by the personality inventory, FPI (Fahrenberg, Selg, \& Hampel, 1978), which measures various 
personality traits. Furthermore, Schandry (1981) also found, that "good perceivers" had higher levels of "state anxiety" than "poor perceivers", as measured by the State Trait Anxiety Inventory Form X-1 (Spielberger, Gorsuch, \& Lushene, 1970). State anxiety refers to "how anxious a person is feeling in a particular moment" (Newham, Westwood, Aplin, \& Wittkowski, 2012, p. 23). In addition, "emotional experience was linked to perception of bodily processes" (Schandry, 1981, p. 488). Finally, Schandry (1981) did not hypothesize about gender differences in terms of interoceptive sensitivity or interoceptive performance.

While scores on the Schandry Task are said to be linked to higher state anxiety within subjects (Schandry, 1981), my intention was to examine the relationship between scores on the Schandry Task with a more widely used measure of anxiety sensitivity, called the Anxiety Sensitivity Index-3 (Taylor et al., 2007), referred to as ASI-3 in this paper. The ASI-3 is a questionnaire intended to measure anxiety sensitivity in terms of four domains. The 18 questions are categorized into Physical Concerns, Cognitive Concerns, Social Concerns, and finally a summary score which constitutes total anxiety sensitivity. Each question (seen in Appendix A, Taylor et al., 2007), is measured on a scale from 0 to 4 measuring how much the participant agrees with each statement, with 0 representing "very little agreement" and 4 indicative of "very much agreement" which translates to "very little anxiety sensitivity" and "very much anxiety sensitivity" respectively. The highest total score that can be obtained is 72 (see scoring in Appendix A, Taylor et al. 2007), and this is indicative of high anxiety sensitivity and the lowest score that can be obtained in 0 , indicative of very low anxiety sensitivity. Taylor et al. (2007) defined anxiety sensitivity as, "the fear of arousal-related sensations, arising from beliefs that the sensations have adverse consequences such as death, insanity, or social rejection" (Taylor et al., 2007, p. 177; Reiss \& McNally, 1985). The study focused on developing an appropriate scale to 
measure anxiety sensitivity as a possible predictor of anxiety disorders (Taylor et al., 2007).

While Taylor et al. (2007) did examine gender in this study to determine the robustness, stability, and fit of the scale, which depicted no gender difference/bias in the use of the ASI-3 questionnaire, they did not report a gender difference for total anxiety sensitivity in comparison to an interoceptive measure of anxiety in such a way as the current study. However, a study by McLean, Asnaani, Litz, \& Hofmann (2011) evaluating the prevalence of anxiety disorders reported that, "women have consistently higher prevalence rates of anxiety disorders than men" (p.1027). Moreover, commenting on this trend, Stoyanova and Hope (2012) stated "[that] despite the well-documented gender effect in anxiety, less is known about contributing factors to women's greater risk for anxiety and fears," (p. 206) suggesting ample reason to explore gender differences in anxiety sensitivity. This is especially interesting because if there is a relationship between the ASI-3 scores of females and their performance on interoceptive tasks, this could be a possible explanation for why females are at a greater risk: because they may be more perceptive of inner sensations.

A new measure of interoceptive awareness called the Mindful Interoception Sampling Task (MIST) has been developed by Dreeben (2012). He describes the MIST in his dissertation proposal entitled, "Measuring Mindful Interoception: Development of the Mindful Interoception Sampling Task.” The MIST is intended to measure mindfulness using an interoceptive task, though using it differently than the Schandry Task. The goal is to have participants sit and listen to a recording (see script in Appendix B, Dreeben, 2012, p. 71), guiding them through focusing their attention on inner sensations in different regions of the body (neck, abdomen, back, and chest) without judgment or distraction. If their mind wanders off task, this is considered "mind wandering," and is recorded by the participant clicking a clicker at the sound of a tone if their 
mind has wandered off task at the sound of the tone, which plays at random throughout the recording. This inaudible click is recorded via software and transmitted to a computer so the researcher can observe. There were 3 tones per 4 body regions, so the maximum total score for mind-wandering a participant can obtain is 12 , indicating a high frequency of mind-wandering (i.e. poor non-judgmental interoception). "Mind-wandering is characteristically described as the interruption of task focus by task-unrelated thought" (Mrazek, Smallwood, \& Schooler, 2012, pg. 442; Smallwood \& Schooler, 2006). This is a good measure because according to Mrazek et al. (2012), "mindfulness and mind-wandering appear to be conceptually opposing constructs with respect to undistracted attention" (p. 443) or "opposite sides of the same coin" (p. 445). Also, the idea is that the MIST will be correlated with low anxiety, while the Schandry Task is correlated with high anxiety (Dreeben, 2012). In other words, while the MIST and the Schandry Task are both interoceptive tasks because they both examine sensations within the body, the MIST is hypothesized to be a measure of mindfulness (Dreeben, 2012), because it examines mindwandering and judgment (both cognitive), while the Schandry Task correlates with state anxiety (Schandry, 1981), because it measures accuracy of physical awareness.

\subsection{Hypotheses}

Using the Schandry Heartbeat Detection Task, the Anxiety Sensitivity Index-3 (ASI-3), and the newly developed MIST, I examined gender differences in anxiety sensitivity and how they may be related to performance on the MIST and Schandry Task, in a sample of participants enrolled in a larger dissertation research project. This research examines whether gender is an important predictor, specifically for anxiety sensitivity, which previous research suggests that "gender specific norms are not necessary [in examining, with regards to the ASI-3 questionnaire in nonclinical settings]" (Osman et al., 2010, p. 50). 
First, I hypothesized that females would score higher than males on the ASI-3. My supposition is that females will report higher levels of anxiety sensitivity than males, based on evidence that females are more prone to anxiety/anxiety disorders than males (McLean et al., 2011); suggesting females may be more likely to interpret certain types of internal sensations as indicative of anxiety. Second, I hypothesized that females would score more accurately (lower) than males on the Schandry Task since evidence suggests that accurate performance on this task (and interoceptive sensitivity overall) is highly correlated with the higher emotional experience (i.e. state anxiety) (Schandry, 1981; Domschke, Stevens, Pfleiderer, \& Gerlach, 2010). Furthermore, this raises interesting questions as to why females may be more anxious; one possibility being that they are more highly attuned than males to internal physical sensations and are more prone to interpret them as signs of anxiety. Finally, I examined gender differences in the newly developed MIST, another interoceptive task. I didn't intend for this to be a formal hypothesis, since there is currently no research using the MIST to investigate mindful interoception. However, I examined performance on the MIST as a function of gender, believing that males might perform with less mind-wandering instances than females, since the MIST is hypothesized to be correlated with low anxiety (Dreeben, 2012). If males perform better, it may be because females are more anxious, and therefore, may not perform mindful interoceptive tasks well, since they may not attend non-judgmentally. The following two tables illustrate the overall predictions for key variables in this study: 
Table 1: Predicted Relationships Among Key Variables for Females

\begin{tabular}{|l|l|l|}
\hline & $\begin{array}{l}\text { Schandry Task: } \\
\text { Total Score }\end{array}$ & $\begin{array}{l}\text { MIST Task: Total } \\
\text { Score }\end{array}$ \\
\hline ASI-3 Total & $\begin{array}{l}\text { High ASI-3 and } \\
\text { Low Schandry } \\
\text { (perform well) }\end{array}$ & $\begin{array}{l}\text { High ASI-3 and } \\
\text { High MIST } \\
\text { (perform poorly) }\end{array}$ \\
\hline
\end{tabular}

Table 2: Predicted Relationships Among Key Variables for Males

\begin{tabular}{|l|l|l|}
\hline & $\begin{array}{l}\text { Schandry Task: } \\
\text { Total Score }\end{array}$ & $\begin{array}{l}\text { MIST Task: } \\
\text { Total Score }\end{array}$ \\
\hline ASI-3 Total & $\begin{array}{l}\text { Low ASI-3 and } \\
\text { High Schandry } \\
\text { (perform poorly) }\end{array}$ & $\begin{array}{l}\text { Low ASI-3 and } \\
\text { Low MIST } \\
\text { (perform well) }\end{array}$ \\
\hline
\end{tabular}




\section{METHOD}

\subsection{Participants}

Participants in this research experiment were students from the University of Louisville. Both females $(n=63)$ and males $(n=13)$ were recruited via the University research website, the SONA Systems. The SONA Systems website targets mainly undergraduate students in the Department of Psychological and Brain Sciences at the University of Louisville; however, to gather a more representative sample, flyers were posted around campus and various classroom presentations were given of the study to recruit a variety of students. The majority of the students were recruited via SONA; however, some participated who were recruited via the alternative methods.

\subsection{Materials}

For the purpose of this study, to monitor heart rate during the different time intervals of the Schandry Task, a ProComp Infinity computerized EKG recording system with surface sensors was used. A stopwatch was used to measure the appropriate time intervals between recordings. Along with the materials needed to replicate the Schandry Task, a randomized numbers generator application was used to determine whether the participant would perform the Schandry Task or the MIST first, and which MIST track the participant would listen to, as the body regions were randomized (the order changed) in each version. The materials needed for the MIST were a recorded script (see Appendix B, Dreeben, 2012, p. 71) delivered via headphones which guided the participants through the mindful interoceptive task. In addition, participants used a response signal 'clicker' and software to keep track of mind wandering instances. 
Furthermore, the ASI-3 Questionnaire (see Appendix A, Taylor et al. 2007) was used to measure rates of anxiety sensitivity.

\subsection{Study Procedure}

Once the prospective participants signed up for a research time slot in SONA Systems or responded to the information regarding the study on a flyer or presentation, they came to the Biobehavioral Research Laboratory where they were met by the experimenter. Before they arrived, however, the researcher determined (using a random numbers generator) whether the participant would complete the MIST or the Schandry Task first. Once in the lab, participants were seated near a computer recording EKG data (the screen was not visible) or the computer with the MIST recording and clicker software (depending on which task they were assigned first). Next, the Informed Consent document was explained and their signature was obtained. Following this, they were permitted to ask questions, and decide whether or not to proceed with the study (the option to withdraw was always available throughout the entirety of the study). They then filled out a demographic information form, after which resting blood pressure and heart-rate were measured. If the Schandry task was administered first, participants were connected to the EKG sensors, and the experimenter made sure that a clear signal was obtained. Subsequently, they completed the second task (either the Schandry or MIST), and sensors were not attached during the MIST. The MIST had 4 versions in which the order of the body region targets was changed. A random numbers generator was used to determine which MIST version a participant would hear. Once the two tasks were completed, participants completed the ASI-3 (see Appendix A, Taylor et al., 2007) questionnaire, still out of direct sight of the experimenter. Finally, the participant was debriefed and dismissed. 
It should be noted that this study is a part of a larger dissertation research project by Dreeben (2012); however, while using the same population, I tested different hypotheses and employed different analyses than those in the main study. The protocol described in this paper is the same as the original study, except for the fact that the larger study includes additional questionnaires which were given at the end of the study in random order along with the ASI-3, determined by the random numbers generator.

\subsection{Schandry Protocol}

The Schandry protocol is similar to the original Schandry Task (Schandry, 1981) and the same as used for all participants in the dissertation study (Dreeben, 2012). Participants were seated next to a computer with the ProComp Infinity EKG software, facing away from the experimenter and the monitor. Then, their wrists were wiped with sanitary alcohol wipes. Next, the electrode sensors were attached to the wrists with two on the left wrist, and one on the right. The transmission cables from the EKG computer were then attached to the sensors; the yellow indicator went on the right wrist, while the black and blue indicators went on the left wrist. Participants were then asked to sit with their feet flat to the ground in front of them, and their arms laying flat on their legs with their wrists facing upward. Subsequently, they were instructed about the three timing intervals, though the interval times were not divulged; they were simply told to count their heartbeats as accurately as possible in each interval. During a 60 second break after the instructions were given, a test heart rate measurement was done to ensure reliable equipment reading. If a clear signal was not obtained, the participant had the option of having a chest placement of the sensors to try to obtain a clearer signal, though this alternative proved to be unnecessary as reliable readings were obtained for all participants with the standard wrist placement. 
Once a satisfactory placement was obtained, on a "go" signal participants counted their heart rate for 25 seconds, then on a "stop" signal, they reported their counted heart rate to the experimenter (during a 30 second break the actual measured heart rate was saved into the recoding software). This was repeated for the 35 , and 45 second intervals. Following this, the participant was disconnected from the physiological recording cables and sensors in order to perform other tasks (the MIST and questionnaires). Scoring the Schandry Task involved taking the absolute value of the reported number of heart beats subtracted by the actual measured number of heartbeats, divided by the actual measured number (percentage error). This was done for each time interval, and the three numbers were summed for a total score. Given that the total score was calculated in terms of percentage error, lower scores indicate more accurate performance.

\subsection{MIST Protocol}

The MIST protocol is identical to the larger dissertation study (Dreeben, 2012). For the MIST, participants were seated next to the computer with the MIST recording and recording software, out of view of the experimenter. The participant was given a hand clicker and headphones so they could listen to the MIST recording. There were 4 different MIST tracks, each with the body region order changed (see table 3). Every participant was first given brief instructions stating that they would listen to a recording and press the clicker intermittently, during the recording at the sound of a tone, if their mind wanders. Further instructions were given on the recording discussing that they were to focus on sensations within their bodies nonjudgmentally and how when the tone rings, if their mind is wandering, they were to hit the clicker, which transmitted to the experimenter where they recorded instances. The full script can be seen in Appendix B (Dreeben, 2012). Within each body region, there were three probe tones 
that rang to randomly throughout the recording measure mind-wandering instances. This means that the total score that can be obtained is 12 , which is indicative of frequent mind-wandering and the lowest possible obtained score is 0 , indicating no mind-wandering. The following table shows the different sequences for the MIST recordings as well as the probe tone timing in each recording sequence:

Table 3: MIST Sequences

\begin{tabular}{|l|l|l|l|l|l|}
\hline & Region I & Region 2 & Region 3 & Region 4 & Total Time \\
\hline PRACTICE TRIAL & \multicolumn{2}{|l|}{ Three probe-triggered ratings x 2 minutes } & \\
\hline & Nose & Nose & Nose & Nose & 2 min. \\
\hline MEASURED TRIAL & Three probe-triggered ratings x 2 min., each region: \\
& Each participant listens to only 1 of 4 following tracks: & \\
\hline Order I track: & Abd. & Chest & Back & Neck & 8 min \\
\hline Order 2 track: & Neck & Abd. & Chest & Back & 8 min \\
\hline Order 3 track: & Back & Neck & Abd. & Chest & 8 min \\
\hline Order 4 track: & Chest & Back & Neck & Abd. & 8 min \\
\hline
\end{tabular}

*Probe timing Nose: 43, 69, and 106 seconds; Abdomen: 20, 70, and100 seconds; Chest: 48, 86, and 107 seconds; Back: 23, 46, and 97 seconds; Neck 50, 72, and 104 seconds. 


\section{RESULTS}

\subsection{Preliminary Analysis}

Data from 75 participants (63 females, 13 males) were included in this study. All of the data analyses were conducted with a $95 \%$ confidence interval and a probability value of .05 . To test the hypotheses involved in this study, I performed independent-samples t-tests, which compares the means of two comparison groups. Initially, however, I tested for normality using Shapiro-Wilk's test for normality of the distributions of each variable. The ASI-3 scores showed a normal distribution for males $(p=.755)$, but not females $(p=.012)$. Furthermore, scores on the Schandry Task were normally distributed for both males $(p=.124)$ and females $(p=.585)$. Finally, the distribution of MIST scores was normal for males $(p=.557)$ but not for females $(p=.004)$. There were no outliers in the distribution of MIST scores for females; however, the ASI-3 distribution had several outliers which, if removed, took out important end-data points. Furthermore, the distributions of both ASI-3 and MIST scores for female samples were slightly positively skewed; however, given that this is an exploratory study, I conducted analyses on the non-normal distributions. An alternative would have been to conduct transformations on the data to achieve a normal distribution. However, after conducting $\log 10$ transformations on the data, which resulted in normalized distributions, statistical tests comparing group means did not yield results that were any different than those obtained using the non-transformed data. Because of this, only results based on non-transformed data are reported below.

\subsection{Descriptive Statistics and Group Statistics}

Data were available for 75 participants overall, 74 (61 females, 13 males) in the ASI-3 sample, 74 (61 females, 13 males) in the Schandry sample, and 75 (62 females, 13 males) in the 
MIST sample. Higher scores on the ASI-3 Questionnaire indicate higher anxiety sensitivity levels. With this in mind, females $(M=23.95)$ scored higher than males $(M=17.54)$ on this measure. Likewise, higher scores on the Schandry Task indicate less accurate performance on the task. With this in mind, females $(\mathrm{M}=1.023)$ performed slightly more accurately on the Schandry Task than did males $(\mathrm{M}=1.092)$. Lastly, higher scores on the MIST indicate increased instances of mind-wandering on the MIST task; with this in mind, males $(\mathrm{M}=5.31)$ performed slightly better (i.e. fewer reported episodes of mind-wandering) than females $(M=6.27)$. This suggests that males were slightly less distracted than females on the MIST, and consequently slightly more attuned to interoceptive signals than females. (See Table 4).

\section{Table 4: Summary Table of Descriptive Statistics}

\begin{tabular}{|l|l|r|r|r|r|r|r|}
\hline Variable & Gender & $\mathrm{N}$ & Mean & $\begin{array}{r}\text { Std. } \\
\text { Deviation }\end{array}$ & $\begin{array}{r}\text { Std. } \\
\text { Error } \\
\text { Mean }\end{array}$ & Minimum & Maximum \\
\hline \multirow{2}{*}{$\begin{array}{l}\text { Schandry Task Total } \\
\text { Score }\end{array}$} & Male & 13 & 1.09 & .766 & .213 & .04 & 2.18 \\
\cline { 2 - 7 } & Female & 61 & 1.02 & .469 & .060 & .03 & 2.13 \\
\hline MIST Total Score & Male & 13 & 5.31 & 2.097 & .582 & 2 & 9 \\
\cline { 2 - 8 } & Female & 62 & 6.27 & 2.457 & .312 & 2 & 12 \\
\hline ASI-3 Total Score & Male & 13 & 17.54 & 8.151 & 2.261 & 5 & 31 \\
\cline { 2 - 8 } & Female & 61 & 23.95 & 10.865 & 1.391 & 7 & 50 \\
\hline
\end{tabular}

*Schandry Task range: $0-x$, where $x$ is percentage error; MIST range: $0-12,3$ data points $\mathrm{x} 4$ regions; ASI-3 range: 0-72, 18 items x 0 - 4 score per item (see Appendix A)

\subsection{Independent Samples T-Test: ASI-3}

To test for differences between female and male participants on the ASI-3 Questionnaire, I performed an independent samples t-test on the data. Levene's Test for Equality of Variances 
indicated that the variance did not differ significantly $(\mathrm{p}=.339)$. A one-tailed (directional) t-test to evaluate the hypothesis that females would have significantly higher total ASI-3 scores than males, resulted in a significant difference in the predicted direction: $t(72)=-2.007 ; p=.02$. The average score for females $(M=23.95)$ was significantly greater than males $(M=17.54)$, indicating higher reported anxiety sensitivity among females.

\subsection{Independent Samples T-Test: Schandry Task}

To test for differences between female and male participants on the Schandry Task, I performed an independent samples t-test. Levene's Test for Equality of Variances indicated that there was significant difference in the variance of the samples $(p=.001)$. Because of the exploratory nature of this study, it was decided to proceed with a one-tailed (directional) t-test for equality of means. It was predicted that females would perform more accurately than males on the Schandry, but the results of the t-test did not support this prediction: $\mathrm{t}(13.976)=.313$; $\mathrm{p}=.38$. This suggests that male $(\mathrm{M}=1.092)$ and female $(\mathrm{M}=1.023)$ accuracy scores did not differ significantly.

\subsection{Independent Samples T-Test: MIST}

To assess whether total MIST scores differed significantly for females and males, I performed an independent samples t-test. This was an exploratory hypothesis, since the MIST is a new measure of interoceptive awareness. The prediction was that males, having lower anxiety sensitivity, would likely perform better than females, in the sense of reporting fewer instances of mind-wandering. Levene's Test for Equality of Variances showed that the sample variances did not differ significantly $(\mathrm{p}=.350)$. I performed a one-tailed (directional) t-test to determine whether males reported fewer mind-wandering instances than females: $t(73)=-1.319, p=.09$. 
Results of this analysis reveal that males $(\mathrm{M}=5.31)$ did not perform significantly better (less mind-wandering) than females ( $M=6.27)$, though there appears to be a trend in the predicted direction. It is possible that the unbalanced sample sizes may have affected the outcome of this analysis. 


\section{DISCUSSION}

This study found support for one of the three hypotheses tested in this study, which involved relationships between anxiety sensitivity and performance on two interoceptive awareness tasks. The first hypothesis predicted that females would have higher reported levels of anxiety sensitivity than males, as measured by the ASI-3, and was supported. It should be noted that average scores on the ASI-3 in the current study ( $M=17.54$ for males, $M=23.95$ for females) were significantly higher overall, than those reported by Osman et al. (2010), $(M=12.57$ for males, $M=13.18$ for females) for a sample of 462 undergraduate students. This suggests that the students in the current sample report higher levels of anxiety sensitivity than a sample of undergraduates from another university setting, raising questions about what differentiates these two populations. Of further note, the 3 subscales of the ASI-3 were compared using independent samples t-tests to assess for possible gender differences. I found no significant difference on the Physical, Cognitive, or Social Concerns subscales; however, both females and males collectively scored highest on the Social Concerns subscale.

The second hypothesis predicted that females would score more accurately (achieving

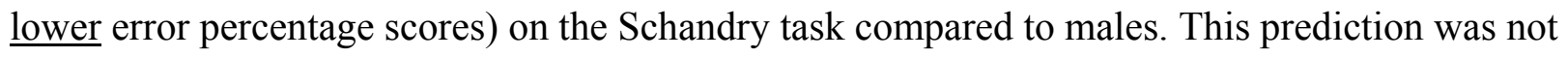
supported, as the means for males and females were found to be similar, suggesting no significant difference. This is interesting, given that Schandry (1981) found that more accurate performance on Schandry task was correlated with state anxiety, self reportedly measured by the State Trait Anxiety Inventory Form X-1 (Spielberger, Gorsuch, \& Lushene, 1970). In the present study though, while the females scored higher on the ASI-3, a measure of anxiety sensitivity, they did not score more accurately on the Schandry task, as predicted. One manifestation of this difference is found in error rate percentages. Schandry (1981) reported that nearly half of the 
participants had error rates of .5 or less, whereas corresponding rates for participants were somewhat higher. The fact that there were more errors overall in the present study may have obscured possible gender-related differences.

Similarly, the third hypothesis, predicting that males would perform with less mindwandering instances on the MIST than females, also failed to achieve support. It is possible that anxiety sensitivity may not be an accurate predictor of mindful interoception, or it may be that there are methodological or participant-related issues that contributed to the absence of predicted contrast. Currently, there are no other measures comparable to the MIST that might help explain this finding. It is interesting to note that anxiety sensitivity is said to measure fear of [internal] sensations (Taylor et al., 2007), yet while women scored higher on the ASI-3, they did not score significantly higher on the MIST, which focuses on internal sensation awareness.

To reiterate, though high scores on the ASI-3 were predicted to correspond with lower (more accurate) scores on the Schandry Task, and high scores (more mind-wandering) on the MIST for females, these relationships were not found. Similarly, males were predicted to score low on the ASI-3, high (less accurate) on the Schandry Task, and low (less mind-wandering) on the MIST, but again, the relationships did not emerge. These findings raise questions about the extent to which anxiety sensitivity may be an accurate predictor of interoceptive awareness.

\subsection{Implications}

The results of this study revealed interesting but ambiguous effects of gender on anxiety sensitivity and interoceptive awareness. First, although females reported higher perceived anxiety sensitivity than males (measured by the ASI-3), they did not perform more accurately on the Schandry task, which reportedly is positively associated with state anxiety (Schandry, 1981). 
One consequence of this is that perhaps the Schandry Task is less strongly linked to anxiety sensitivity than it is to anxiety per se.

Furthermore, although this study found a significant difference between females and males in terms of anxiety sensitivity, that difference does not appear to affect how they performed on either of the interoceptive tasks employed in this study. These results leave unanswered questions about why the apparent difference in anxiety sensitivity did not translate into differential performance on the interoceptive awareness tasks. Perhaps anxiety sensitivity does not play a significant role in interoceptive awareness. Perhaps methodological shortcomings may contribute to these findings. It may also be that a different population — persons with anxiety disorders, for example—would yield different results.

It is important to note that both males and females reported higher scores on the Social Concerns anxiety sensitivity subscale of the ASI-3 than on either of the two other subscales. Social Concerns are said to be "associated with the belief that publically observable anxiety reactions will elicit social rejection or ridicule" (Taylor et al., 2007, p. 177). It would be interesting to see if this pattern holds for other groups (e.g. non-student) as well. The relative impact of this facet of anxiety sensitivity may somehow account for why the ASI-3 yielded gender differences in anxiety sensitivity, yet not for interoceptive task performance. Perhaps a population that scored higher on the physical subscale of anxiety sensitivity (medical patients or trauma victims, for example), might reveal a stronger association with interoceptive task performance, since interoception is predominately a measure of inner physical states, according to Craig (2003). 


\subsection{Strengths and Limitations}

There are several limitations to this study which may affect its validity. For example, the sample sizes were not comparable (62 females, 13 males). There were significantly more females than males in the sample; an apparent reflection of the undergraduate subject pool from which they were drawn. This inequality may have affected the results of the independent samples t-tests performance to test the hypotheses for this study, especially those involving the Schandry and the MIST tests. Another factor that is somewhat of a limitation is the fact that the sample was comprised of undergraduate students from a single university setting, which limits generalizability to other student and non-student populations. Both of these factors may have increased the likelihood of a type two error (the failure to reject a false null hypothesis) in the analysis of the MIST and the Schandry Task data.

Strengths of this study include its novel use of gender as a key variable to evaluate anxiety sensitivity, and its possible impact on interoceptive task performance. Furthermore, this study employed two widely used measures of both anxiety sensitivity (the ASI-3) and interoceptive awareness (the Schandry Task). Finally, this study employed a novel, but promising, measure of mindful interoception (the MIST) to compare performance of males and females.

\subsection{Future Research}

It would be beneficial to conduct further research to determine whether, and in what ways, females are more anxious than males. The current study did support the hypothesis that females would report higher levels of anxiety sensitivity, but this did not appear to affect their performance on the Schandry task, which has been reported to correlate with state anxiety 
(Schandry, 1981). Also, future research could compare the ASI-3 measures of anxiety that do not use the self-report format— physiological variables, for example—which would allow researchers to differentiate perceived versus objectively measured correlates of anxiety. In a similar manner, it would be instructive to investigate the relationship between performance on the Schandry Task and other anxiety measures to determine how assessment procedures may affect the relationship.

One additional avenue of possible research would be to see if there are differences in anxiety sensitivity and performance on interoceptive awareness tasks related to demographic variables other than gender. This might offer insight into what factors underlie the capacity for mindfulness and interoceptive sensitivity, and possible conclusions as to why this may be. If anxiety sensitivity is in fact not a significant predictor of interoceptive sensitivity, then what is? This is an important issue to address as research in the area of interoceptive awareness continues to grow.

Finally, looking back on the gender and sex differences discussed in the introduction, future research can help determine biological and sociocultural contributions to differential performance on anxiety sensitivity measures and interoceptive sensitivity tasks. For instance, while males did perform with less mind-wandering instances on the MIST and were overall less accurate on the Schandry Task, their performance did not differ from females in a statistically significant manner. Perhaps a more refined way of assessing either biological or sociocultural factors related to gender might provide a more sensitive and accurate way to differentiate performance. 


\section{Acknowledgments:}

I would like to thank my mentor, Dr. Paul Salmon, graduate student Sam Dreeben, and my committee members Drs. Tamara Newton and Karen Hadley, for their guidance and support throughout the process of writing this thesis. 


\section{REFERENCES}

Ainley, V., Tajadura-Jiménez, A., Fotopoulou, A., \& Tsakiris, M. (2012). Looking into myself: Changes in interoceptive sensitivity during mirror self-observation. Psychophysiology, 49(11), 1672-1676. doi:10.1111/j.1469-8986.2012.01468.x

Craig, A. (2003). Interoception: the sense of the physiological condition of the body. Current Opinion In Neurobiology, 13(4), 500. doi:10.1016/S0959-4388(03)00090-4

Doğan, O. (2012). The etiology of anxiety disorders. Anatolian Journal Of Psychiatry / Anadolu Psikiyatri Dergisi, 13(3), 224-231.

Domschke, K., Stevens, S., Pfleiderer, B., \& Gerlach, A. L. (2010). Interoceptive sensitivity in anxiety and anxiety disorders: An overview and integration of neurobiological findings. Clinical Psychology Review, 30(1), 1-11. doi:10.1016/j.cpr.2009.08.008

Dreeben, J. Samuel. (2012). Measuring Mindful Interoception: Development of the Mindful Interoception Sampling Task (MIST). Dissertation Proposal: 1-84.

Fahrenberg, J., Selg, H., \& Hampel, R. (1978) Das Freiburger Persönlichkeitsinventar, FPI. Göttingen: Verlag für Psychologie, Dr. C.J. Hogrefe.

Kabat-Zinn, J. (2003) Mindfulness-based interventions in context: Past, present, and future. Clinical Psychology, Science and Practice 10, 144-156.

Kang, C., \& Whittingham, K. (2010). Mindfulness: A dialogue between Buddism and clinical psychology. Mindfulness, 1(3), 161-173. doi:10.1007/s12671-010-0018-1 
Keune, P. M., \& Forintos, D. (2010). Mindfulness meditation: A preliminary study on meditation practice during everyday life activities and its association with well-being. Psihologijske Teme, 19(2), 373-386.

Kimura, D. (2000). Sex and cognition. Cambridge: MIT.

McLean, C. P., Asnaani, A., Litz, B. T., \& Hofmann, S. G. (2011). Gender differences in anxiety disorders: Prevalence, course of illness, comorbidity and burden of illness. Journal Of Psychiatric Research, 45(8), 1027-1035. doi:10.1016/j.jpsychires.2011.03.006

Mrazek, M. D., Smallwood, J., \& Schooler, J. W. (2012). Mindfulness and mind-wandering: Finding convergence through opposing constructs. Emotion, 12(3), 442-448. doi:10.1037/a0026678

Muehlenhard, C., \& Peterson, Z. (2011). Distinguishing between sex and gender: History, current conceptualizations, and implicateons. Sex Roles, 64, 791-803. doi:10.1007/s11199-011-9932-5.

Newham, J. J., Westwood, M., Aplin, J. D., \& Wittkowski, A. (2012). State-Trait Anxiety Inventory (STAI) scores during pregnancy following intervention with complementary therapies. Journal Of Affective Disorders, 142(1-3), 22-30. doi:10.1016/j.jad.2012.04.027

Osman, A., Gutierrez, P.M., Smith, K., Qijuan, F., Lozano, G., \& Devine, A. (2010). The Anxiety Sensitivity Index-3: Analyses of Dimensions, Reliability Estimates, and Correlates in Nonclinical Samples. Journal Of Personality Assessment, 92(1), 45-52. doi:10.1080/00223890903379332

Reilly, D. (2012). Exploring the science behind sex and gender differences in cognitive abilities. Sex Roles, 67(3-4), 247-250. doi:10.1007/s11199-012-0134-6 
Reiss, S., \& McNally, R. J. (1985). Expectancy model of fear. In S. Reiss \& R. R. Bootzin (Eds.), Theoretical issues in behavior therapy (pp.107-121). San Diego, CA: Academic Press.

Schandry, R. (1981). Heart beat perception and emotional experience. Psychophysiology, 18(4), 483-488. doi:10.1111/j.1469-8986.1981.tb02486.x

Smallwood, J., \& Schooler, J. W. (2006). The restless mind. Psychological Bulletin, 132(6), 946958. doi:10.1037/0033-2909.132.6.946

Soysa, C. K., \& Wilcomb, C. J. (2013). Mindfulness, self-compassion, self-efficacy, and gender as predictors of depression, anxiety, stress, and well-being. Mindfulness, doi:10.1007/s 12671-013-0247-1

Spielberger, C.D., Gorsuch, R.L, \& Lushene, R.E. (1970) STAI manual for the State-Trait Anxiety Inventory. Palo Alto: Consulting Psychologists Press, Inc.

Stoyanova, M., \& Hope, D. A. (2012). Gender, gender roles, and anxiety: Perceived confirmability of self report, behavioral avoidance, and physiological reactivity. Journal Of Anxiety Disorders, 26(1), 206-214. doi:10.1016/j.janxdis.2011.11.006

Taylor, S. (1999). Anxiety sensitivity: Theory, research, and treatment of the fear of anxiety. Mahwah, NJ: Erlbaum.

Taylor, S., Zvolensky, M. J., Cox, B. J., Deacon, B., Heimberg, R. G., Ledley, D., \& ... Cardenas, S. (2007). Robust dimensions of anxiety sensitivity: Development and initial validation of the Anxiety Sensitivity Index-3. Psychological Assessment, 19(2), 176-188. doi:10.1037/1040-3590.19.2.176 
Appendix A: The Anxiety Sensitivity Index-3 Questionnaire (Taylor et al. 2007)

Please circle the number that best corresponds to how much you agree with each item. If any items concern something that you have never experienced (e.g., fainting in public) answer on the basis of how you think you might feel if you had such an experience. Otherwise, answer all items on the basis of your own experience. Be careful to circle only one number for each item and please answer all items.

\section{0 $=$ Very Little $\quad 1=$ A Little $\quad 2=$ Some $\quad 3=$ Much $\quad 4=$ Very Much}

1. It is important for me not to appear nervous.

$$
\begin{array}{lllll}
0 & 1 & 2 & 3 & 4
\end{array}
$$

2. When I cannot keep my mind on a task, I worry that I might be going crazy.

$\begin{array}{lllll}0 & 1 & 2 & 3 & 4\end{array}$

3. It scares me when my heart beats rapidly.

$$
\begin{array}{llllll}
0 & 1 & 2 & 3 & 4
\end{array}
$$

4. When my stomach is upset, I worry that I might be seriously ill.

$$
\begin{array}{lllll}
0 & 1 & 2 & 3 & 4
\end{array}
$$

5. It scares me when I am unable to keep my mind on a task.

$\begin{array}{lllll}0 & 1 & 2 & 3 & 4\end{array}$

6. When I tremble in the presence of others, I fear what people might think of me.

$$
\begin{array}{llllll}
0 & 1 & 2 & 3 & 4
\end{array}
$$

7. When my chest feels tight, I get scared that I won't be able to breathe properly.

$$
\begin{array}{lllll}
0 & 1 & 2 & 3 & 4
\end{array}
$$

8. When I feel pain in my chest, I worry that I am going to have a heart attack.

$$
\begin{array}{lllll}
0 & 1 & 2 & 3 & 4
\end{array}
$$

9. I worry that other people will notice my anxiety.

$$
\begin{array}{lllll}
0 & 1 & 2 & 3 & 4
\end{array}
$$

10. When I feel "spacey" or spaced out I worry that I may be mentally ill.

$\begin{array}{lllll}0 & 1 & 2 & 3 & 4\end{array}$


11. It scares me when I blush in front of people.

$$
\begin{array}{lllll}
0 & 1 & 2 & 3 & 4
\end{array}
$$

12. When I notice my heart skipping a beat, I worry that there is something seriously wrong with me.

$\begin{array}{lllll}0 & 1 & 2 & 3 & 4\end{array}$

13. When I begin to sweat in a social situation, I fear people will think negatively of me.

$\begin{array}{lllll}0 & 1 & 2 & 3 & 4\end{array}$

14. When my thoughts seem to speed up, I worry that I might be going crazy.

$\begin{array}{lllll}0 & 1 & 2 & 3 & 4\end{array}$

15. When my throat feels tight, I worry that I could choke to death.

$\begin{array}{lllll}0 & 1 & 2 & 3 & 4\end{array}$

16. When I have trouble thinking clearly, I worry that there is something wrong with me.

$\begin{array}{lllll}0 & 1 & 2 & 3 . & 4\end{array}$

17. I think it would be horrible for me to faint in public.

$$
\begin{array}{llllll}
0 & 1 & 2 & 3 & 4
\end{array}
$$

18. When my mind goes blank, I worry there is something terribly wrong with me.

$\begin{array}{lllll}0 & 1 & 2 & 3 & 4\end{array}$

Scoring the ASI-3:

Physical Concerns subscale prompts: 3, 4, 7, 8, 12, 15

Cognitive Concerns subscale prompts: 2, 5, 10, 14, 16, 18

Social Concerns subscale prompts: $1,6,9,11,13,17$

Sum each subscale for subscale scores. Add subscales for total score. 
Appendix B: The MIST Script (Dreeben, 2012, p. 71)

In a moment, you will begin a guided attention task. If you choose, you can close your eyes to better focus on the task, or if you prefer, you can leave your eyes open.

During this task, you will be asked to direct your attention to inner sensations within different regions of your body. As you attend to these regions of the body you may notice any number of inner sensations. Some people notice sensations such as tightness, looseness, coolness, warmth,or tingling. It does not matter which sensation you attend to, or whether it's a strong sensation or a weak sensation. Just focus on the sensations in your body as they are. (Brief pause....)

Now...in addition to inner physical sensations, your attention may also be drawn from time to time to thoughts or other mental events that come and go...we call this 'mind wandering', and it's a very common experience. Examples of mind wandering include internal conversations, daydreaming, or becoming distracted by a smell or sound.

During the task you may find that instances of mind wandering draw your attention away from the physical sensations you are experiencing in the present moment. Whenever this occurs, we ask that you refocus your attention on the internal sensations present in your body in that moment.

Throughout the task, you will periodically hear a signal that sounds like this: (tone). At that moment, we ask that you determine if your attention is focused on inner physical sensations or on something else, like thoughts. If your attention has wandered from physical sensations, please press the clicker in your hand. If your attention is presently on inner sensations, please continue 
focusing on the sensations and do not press the clicker. Other than pressing the clicker, you will not need to move your body during the task.

During this task, you may have thoughts about the sensations in your body (for example, 'I wonder if my stomach feels queasy because of the food I ate for breakfast"). Although these thoughts are related to the body, they have still shifted the attention away from sensations that are occurring in that moment. If you notice that you are having thoughts about inner sensations rather than focusing on the sensations themselves, consider this 'mind wandering'. It is important that you understand this distinction so that you are able to make the most accurate reports possible.

Before we practice, please let the facilitator know if you have any questions about this distinction or more generally about the task. (pause)

Okay, let's practice...now bringing your attention to the inside of the nose....just being aware of whatever inner sensations you are experiencing. ( 2 minutes of silence with 3 signals)

Please press the clicker to indicate that you are awake. Thank you.

At this time, please let your facilitator know if you have any further questions. (pause) Remember it is very important that you report instances of mind wandering as truthfully as possible. Thank you for your participation - let's begin. (pause)

Now bringing your attention to the abdomen, the center of the body....just being aware of whatever inner sensations you are experiencing. ( 2 minutes of silence with 3 signals)

Please press the clicker to indicate that you are awake. Thank you. 
Now, bringing your attention to the chest region of the body... just being aware of whatever inner sensations you are experiencing ( 2 minutes of silence with 3 signals)

Please press the clicker to indicate that you are awake. Thank you.

Now, bringing your attention to the muscles in your back... just being aware of whatever inner sensations you are experiencing ( 2 minutes of silence with 3 signals)

Please press the clicker to indicate that you are awake. Thank you.

Now, bringing your attention to the neck and throat... just being aware of whatever inner sensations you are experiencing ( 2 minutes of silence with 3 signals)

Please press the clicker to indicate that you are awake. Thank you.

This marks the end of this guided attention task. Please take your time redirecting your attention outwardly. 